\title{
Diagnostic accuracy of ultrasonography for the prenatal diagnosis of esophageal atresia and tracheoesophageal fistula
}

\author{
CONG WANG $^{1}$, XIAOFEI NING ${ }^{2}$, YANGCAN DUAN $^{1}$, ZHONGLU ZHANG $^{1}$ and SHAOCHUN WANG ${ }^{1}$ \\ Departments of ${ }^{1}$ Ultrasonography and ${ }^{2}$ Gastrointestinal Surgery, \\ Affiliated Hospital of Jining Medical University, Jining, Shandong 272000, P.R. China
}

Received August 4, 2020; Accepted March 19, 2021

DOI: $10.3892 /$ etm.2021.10075

\begin{abstract}
Ultrasound is recommended as a first-line requirement prior to MRI or amniotic fluid analysis, which have high diagnostic accuracy for esophageal atresia (EA). Therefore, the aim of the present prospective study was to evaluate the accuracy of high-performance ultrasound for the prenatal examination of EA/tracheoesophageal fistula (TOF). In total, 64 pregnant women with fetuses suspected of having EA/TOF participated in the study. The gestational age of the fetuses ranged between 16 and 40 weeks, with a mean of $26.33 \pm 3.57$ weeks. Ultrasound images of the esophagus and trachea on parasternal and para-aortic axis longitudinal and transverse sections were compared with the results of standard postnatal diagnostic tests. Sensitivity and specificity values were determined and a receiver operating characteristic (ROC) curve was generated. Among all the fetuses screened, 16 were suspected of having EA/TOF during the prenatal ultrasonography. In postnatal examinations, 34 cases of EA/TOF were confirmed, corresponding to an EA/TOF incidence of $53.2 \%$ (95\% CI, 40.2-65.7\%). The area under the ROC curve (AUC) was lower for prenatal ultrasonography compared with postnatal diagnostic tests $(\mathrm{AUC}=0.55 ; 95 \% \mathrm{CI}$, 0.44-0.65). Considering postnatal examination as the gold standard, prenatal ultrasonography had a sensitivity of $29.4 \%$ (95\% CI, 15.1-47.5\%) and a specificity of $80 \%$ (95\% CI, 61.4-92.3\%) for the diagnosis of EA/TOF. In addition, the positive predictive value was $62.5 \%$ (95\% CI, 35.4-82.8\%), the negative predictive value was $50 \%$ (95\% CI, 35.2-64.8\%), the positive likelihood ratio was 1.47 (95\% CI, 0.61-3.56) and the negative likelihood ratio was 0.88 (95\% CI, 0.67-1.17). The results of the present study indicate that preoperative ultrasound has poor sensitivity but very good specificity for the diagnosis of EA/TOF. The use of ultrasound alone would
\end{abstract}

Correspondence to: Dr Yangcan Duan, Department of Ultrasonography, Affiliated Hospital of Jining Medical University, 89 Guhuai Road, Jining, Shandong 272000, P.R. China

E-mail: duanyangcan1104@163.com

Key words: esophageal atresia, high-performance ultrasound, prenatal diagnosis, fetus result in a high rate of a false-positive diagnoses. However, prenatal ultrasonography may be useful as a preliminary screening tool to exclude patients for suspected EA/TOF.

\section{Introduction}

Esophageal atresia (EA) is a congenital malformation characterized by a gap in the esophagus, which ends in a closed pouch and is not able to deliver food or saliva to the stomach. Most commonly, instead of a simple disruption, the defect presents as an abnormal connection between the esophagus and the trachea known as a tracheoesophageal fistula (TOF) (1). EA with or without TOF remains the most common congenital anomaly of the esophagus, and approximately half of the affected fetuses present associated complications on the vertebrae, heart, kidneys, limbs and the digestive or urinary systems (2). It has a prevalence of $\sim 2.44$ per 10,000 births according to the pooled analysis of 18 international birth defect surveillance programs (3).

Improvements achieved in the survival of patients with EA $(\sim 90 \%)$ can be largely attributed to advances in neonatal intensive care, including prompt surgical interventions, which in turn depend on early diagnosis (4). EA is most commonly diagnosed during the first $24 \mathrm{~h}$ of life, but may also be detected at other times either pre- or postnatally $(5,6)$. At birth, pediatricians check the patency of the newborn's esophagus by carefully introducing a nasogastric probe into the stomach. In cases of atresia, it is not possible to advance the probe more than a few centimeters into the esophagus; however, visualization of the malformation per se and determination of the type and location of any tracheoesophageal fistulas require radiography of the abdomen. The diagnosis of EA is rarely confirmed before birth as it requires the use of other approaches, such as ultrasound, amniotic fluid analysis or nuclear magnetic resonance imaging (MRI) to study the organs in more detail (6).

Esophageal defects are typically located at the level of the cervicothoracic junction, and assessing this particular area of interest is challenging due to the shadow cones generated by bony structures, including the dorsal cervical spine and clavicles. Routine ultrasound examinations are not able to present the normal esophagus because of the similarity of its collapsed lumen and tissue textures with those of surrounding organs. Hence, ultrasound alone has been considered an 
unsatisfactory diagnostic tool for the identification of EA prenatally due to a high rate of false-positive diagnoses; however, a suspicious ultrasound is recommended as a first-line requirement prior to MRI or amniotic fluid analysis, which have high diagnostic accuracies for EA (7). Accordingly, the present study aimed to perform a methodological re-exploration of the accuracy of ultrasonographic features for the prenatal diagnosis of esophageal atresia and tracheoesophageal fistula. High-performance ultrasound with parasternal and para-aortic axis longitudinal and transverse sections was used.

\section{Materials and methods}

Informed consent and ethical approval. The Ethics Committee of the Affiliated Hospital of Jining Medical University approved the study protocol (approval no. JYFY-2019-17). All pregnant women gave their informed consent prior to the examinations. The study was conducted following the tenets of the Declaration of Helsinki.

Study design, setting and participants. The study was prospectively conducted in the Affiliated Hospital of Jining Medical University (Jining, China), a public health institution of higher education learning in Shandong province. The participants were recruited at the Affiliated Hospital of Jining Medical University and local referral centers from January 2014 to June 2018.

Inclusion criteria for the study were as follows: Pregnant women whose fetuses were suspected of presenting with EA/TOF based on high-performance ultrasonic detection, and an amniotic fluid index $>25 \mathrm{~cm}$ or a fetus with no or small stomach. Patients who did not provide informed written consent were excluded.

Study procedure. A GE Voluson E8 or E10 ultrasonic diagnostic apparatus (GEHealthcare) with a probe frequency of 2.5-5.0 MHz was used to perform the examinations. Following a routine fetal ultrasound and biological measurements, longitudinal and transverse views were used to continuously check the esophagus and trachea. Esophageal and tracheal images were obtained from patients in the supine position (Fig. 1) and prone position (Figs. 2 and 3). The standard for esophageal soft tissue thickening was considered to be that the thickness of the esophagus, measured on both surfaces of the section, was larger than the diameter at the beginning of the left subclavian artery during the systolic period of the long axis of the aortic arch.

Partial echo interruption of the wall and esophageal fistula were considered as screening indicators for suspected EA. The cases were classified as presenting signs suspicious of EA based on findings in the tracheal main bronchial tree (dilated diameter of the tracheal main bronchial tree), the presence or absence of TOF, the length of the atresia and other complications. The shape of the esophagus was evaluated to assess the severity of EA. Features of the suspected cases were compared with the results of the postpartum autopsy, surgery or other examination results to confirm the diagnosis and to assess the diagnostic accuracy of the ultrasonographic method.

Statistical analysis. The data were analyzed using STATA software, version 14.2 (StataCorp LP). Continuous variables

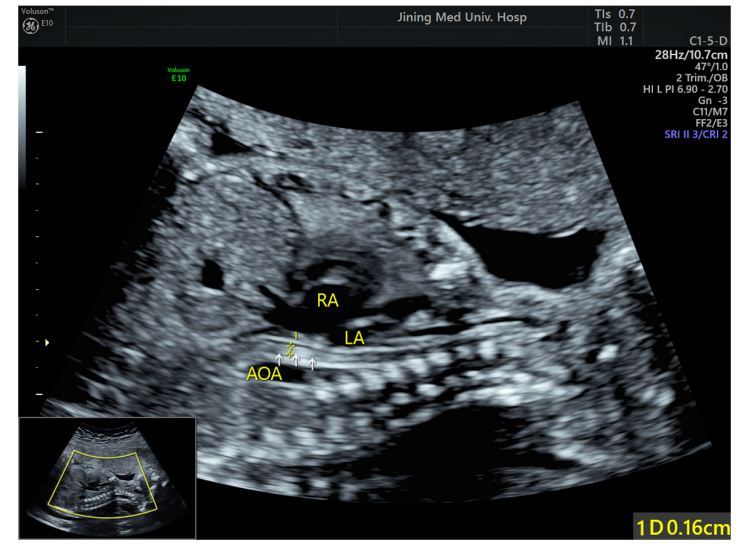

Figure 1. Normal esophagus of a fetus in a supine position. The arrows indicate the esophagus and calipers 1 indicate the diameter of the esophagus. AOA, aorta; LA, left atrium; RA, right atrium.

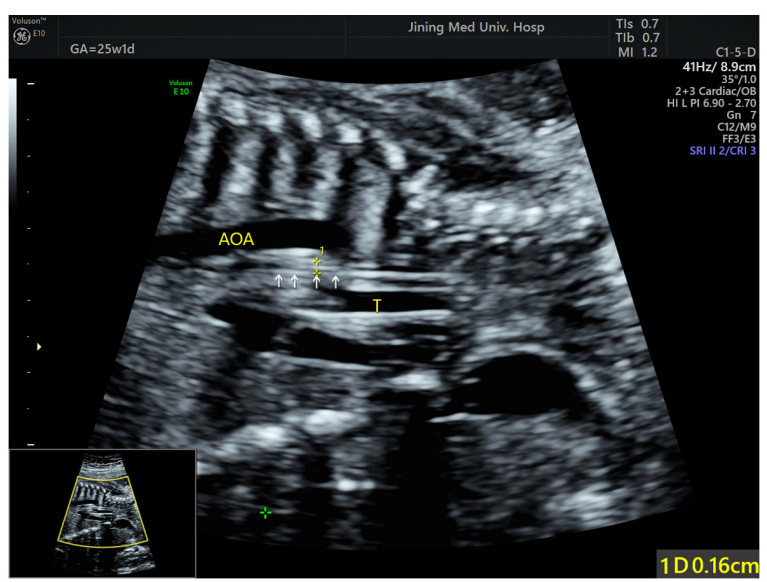

Figure 2. Normal unfilled esophagus of a fetus in a prone position. The arrows indicate the esophagus and calipers 1 shows the diameter of the unfilled esophagus. AOA, aorta; T, trachea.

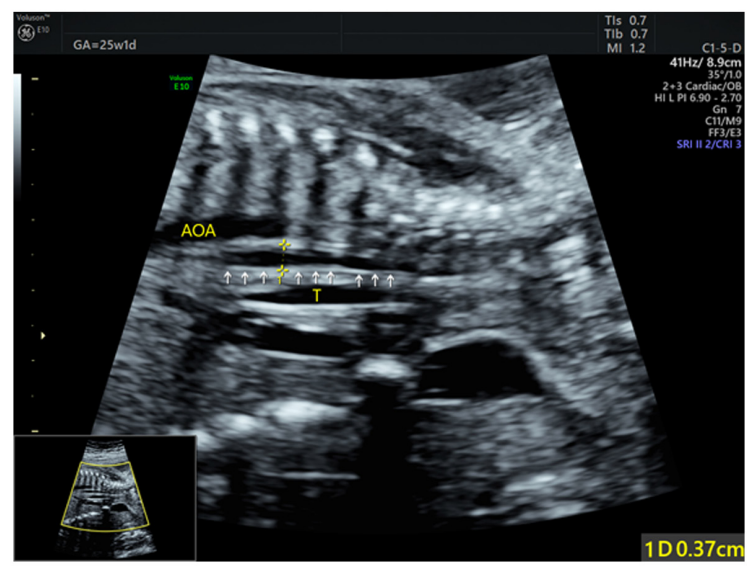

Figure 3. Normal filled esophagus of a fetus in a prone position. The arrows indicate the esophagus and calipers 1 show the diameter of the filled esophagus. AOA, aorta; T, trachea.

are presented as the mean \pm standard deviation (SD). Categorical variables are presented as proportions, with a 95\% confidence interval (CI). Cohen's kappa index was used 
to detect significant agreements between the prenatal and postnatal diagnoses of EA/TOF. The diagnostic accuracy of the prenatal ultrasonography was assessed by calculating the sensitivity, specificity, positive predictive value and negative predictive value based on the gold standard of postnatal examination. In addition, positive and negative likelihood ratios were calculated. A non-parametric estimation of the receiver operating characteristic (ROC) curve was performed to obtain the area under the curve (AUC).

\section{Results}

Prenatal ultrasound results. In total, 64 pregnant women were screened for EA/TOF. The women were between 18 and 42 years old (mean $\pm \mathrm{SD}, 33.24 \pm 3.22$ years). The gestational age of the fetuses was between 16 and 40 weeks (mean \pm SD, $26.33 \pm 3.57$ weeks) (Table I). After obtaining esophageal images through the longitudinal and/or para-aortic axis and transverse sections with the high-frequency ultrasound (Figs. 1-3), concordance was observed between the prenatal and postnatal estimates of the lengths of the esophageal defects

Among all the women screened, 16 were suspected of carrying fetuses with EA/TOF during the prenatal ultrasonography. In total, 34 cases of EA/TOF were confirmed among the 49 postnatal examinations, which corresponded to an EA/TOF incidence of 53.2\% (95\% CI, 40.2-65.7\%). Among the remaining 15 fetuses, certain did not exhibit symptoms for suspected EA, such as feeding difficulty, vomiting or bucking, and the follow-up of certain fetuses was lost.

Statistical analysis results. A weak agreement was detected between the results of the prenatal and postnatal examinations (agreement, 53.2\%; Cohen's kappa=0.10, $\mathrm{P}=0.19$ ). To determine the ability of prenatal ultrasonography to correctly diagnose EA/TOF, its diagnostic accuracy was determined using an ROC curve with postnatal examination as the gold standard (Fig. 4). Table II shows the results of the diagnostic accuracy test for prenatal ultrasonography. The AUC was lower for prenatal ultrasonography compared with postnatal diagnostic tests (AUC $=0.55 ; 95 \% \mathrm{CI}, 0.44-0.65)$.

In further analysis using postnatal examination as the gold standard, prenatal ultrasonography was demonstrated to have a sensitivity of $29.4 \%$ (95\% CI, $15.1-47.5 \%$ ), a specificity of $80 \%$ (95\% CI, 61.4-92.3\%), a positive predictive value of $62.5 \%$ (95\% CI, 35.4-82.8\%) and a negative predictive value of $50 \%$ (95\% CI, 35.2-64.8\%). The positive likelihood ratio was 1.47 (95\% CI, 0.61-3.56) and the negative likelihood ratio was 0.88 (95\% CI, 0.67-1.17).

Ultrasonography revealed various changes in the esophageal region of the fetuses. The main changes revealed by the ultrasonography included thickened esophageal soft tissue (Fig. 5), a fistula between the esophagus and trachea (Fig. 6) and changes in the diameter at the beginning of the left subclavian artery (Fig. 7). The diameter in the beginning of the left subclavian artery was not altered. The thickness of the esophageal soft tissue was compared with the diameter in the beginning of the left subclavian artery. The standard for esophageal soft tissue thickening was considered to be that the thickness of the esophagus, measured on both surfaces of the section, was larger than the diameter at the beginning of
Table I. Characteristics of the study participants $(n=64)$.

\begin{tabular}{lc}
\hline Characteristics & Data \\
\hline Age of the mother, years & $33.24 \pm 3.22$ \\
Gestational age of the fetus, weeks & $26.33 \pm 3.57$ \\
EA/TOF incidence, n (\%) & \\
Present & $34(53.2)$ \\
Absent & $30(46.8)$ \\
\hline
\end{tabular}

Ages are presented as the mean \pm standard deviation. EA, esophageal atresia; TOF, tracheoesophageal fistula.

Table II. Measurements of diagnostic accuracy between prenatal ultrasonography and postnatal examinations $(n=64)$.

\begin{tabular}{lr} 
Characteristics & Diagnostic accuracy (95\% CI) \\
\hline Area under the curve & $0.55(0.44-0.65)$ \\
Sensitivity, $\%$ & $29.4(15.1-47.5)$ \\
Specificity, \% & $80(61.4-92.3)$ \\
Positive predictive value, $\%$ & $62.5(35.4-82.8)$ \\
Negative predictive value, $\%$ & $50(35.2-64.8)$ \\
Positive likelihood ratio & $1.47(0.61-3.56)$ \\
Negative likelihood ratio & $0.88(0.67-1.17)$ \\
\hline
\end{tabular}

CI, confidence interval.

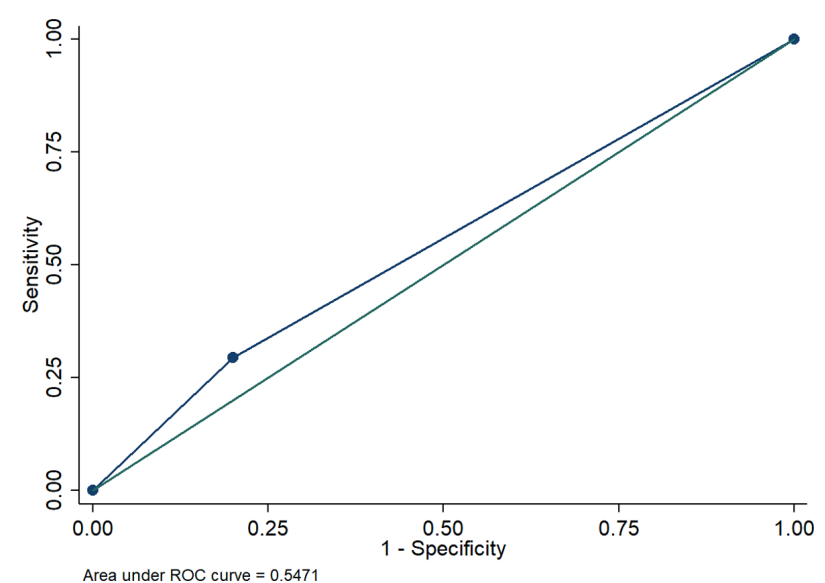

Figure 4. ROC curve showing the sensitivity and specificity of prenatal ultrasonography and postnatal examination. ROC, receiver operating characteristic.

the left subclavian artery during the systolic period of the long axis of the aortic arch.

\section{Discussion}

Improving the diagnostic accuracy of prenatal EA assessments is challenging due to the presence of associated malformations and the limited visibility of the structures $(8,9)$. Therefore, the present study was performed to assess the diagnostic accuracy 


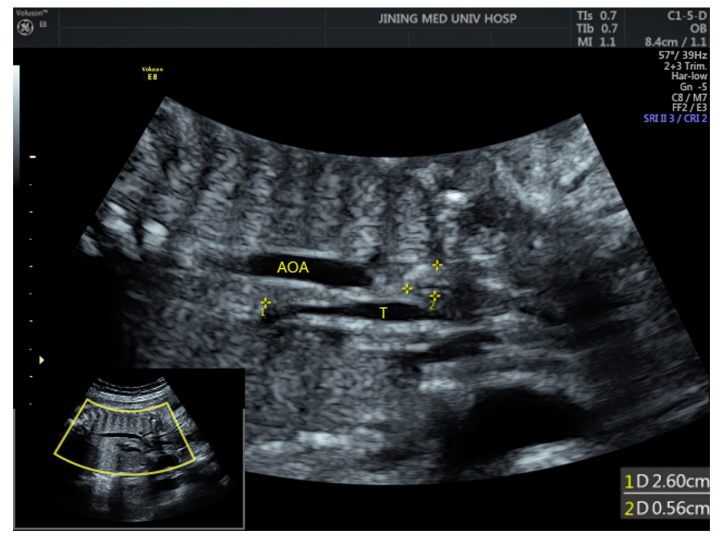

Figure 5. Thickened esophageal soft tissue and length of the atresia in a case of EA. Calipers 1 indicate the atresia and calipers 2 indicate the thickened esophageal tissue. AOA, aorta; T, trachea.

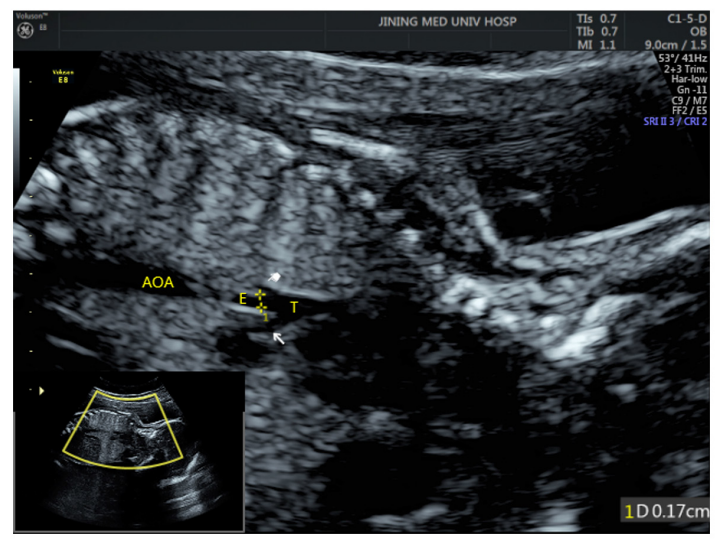

Figure 6. Fistula between the esophagus and the trachea. Calipers 1 indicate the fistula, the pointing hand indicates the left main bronchus, and the arrow indicates the right main bronchus. AOA, aorta; T, trachea; E, esophagus.

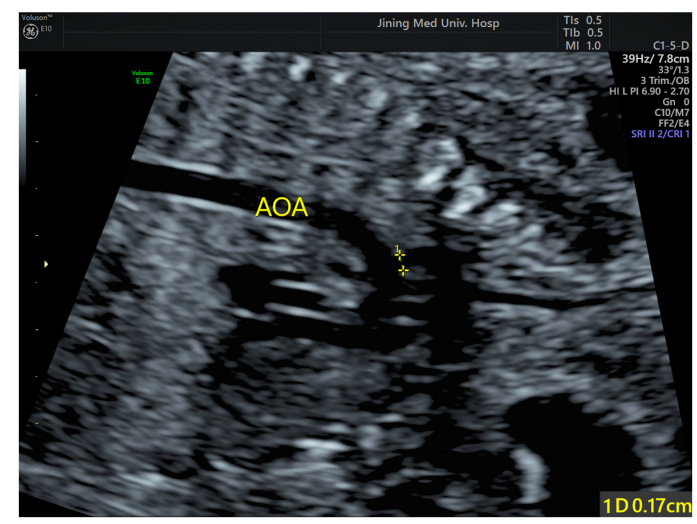

Figure 7. Measurement of the diameter at the beginning of the left subclavian artery. Calipers 1 indicate the site of measurement. AOA, aorta.

of prenatal ultrasonography for the identification of suspected $\mathrm{EA} / \mathrm{TOF}$

A weak agreement was detected between the findings of prenatal ultrasonography and postnatal examinations in patients with EA/TOF. In addition, prenatal ultrasonography was demonstrated to have poor sensitivity for the identification of EA/TOF and moderate specificity for ruling them out. A similar study conducted by Bradshaw et al (10) in 2016 also reported that prenatal ultrasonography has poor sensitivity $(<30 \%)$, but high specificity at $99 \%$. The low sensitivity may be attributed to the level of expertise of the health professionals performing the procedure. The previous study reported that the sensitivity of ultrasonography for the diagnosis of EA/TOF increased by almost half when the procedure was performed in a specialist center by professionals having a high level of expertise (10). Another previous study, a prospective study involving the examination of 60 fetuses at 19-25 weeks of gestation, attempted to identify the rates of visualization of the normal esophagus using a high-resolution linear transducer. The study reported that complete visualization of the normal esophagus was achieved in $86.7 \%$ of cases and at least partial visualization was achieved in $96.7 \%$ of cases. The study concluded that ultrasound alone is a poor diagnostic tool for the identification of EA prenatally and has a high rate of false-positive diagnoses (7). However, other studies have also demonstrated that direct or indirect sonographic assessments of the esophagus in fetuses suspected of having EA improve the specificity of the diagnosis and prenatal evaluation $(11,12)$.

Despite their limitations, preoperative ultrasound findings continue to serve a vital role in clinical practice as they are necessary for planning the surgical strategy. A study conducted by Su et al (11) in 2014 concluded that preoperative scan findings were useful in $25.0 \%$ of patients. In their study, two infants were treated via a primary cervical approach instead of a thoracotomy, and two infants who were originally misdiagnosed due to stretched distal esophagi that extended upward along the trachea into the proximal pouch did not require any additional strategies.

Ultrasound technology lacks sensitivity for the detection of EA/TOF, despite its usefulness for the prenatal screening of other malformations, including congenital heart disease, central nervous system anomalies, skeletal deformities and gastrointestinal tract malformations such as a dilated cecum, which is a potential ultrasound sign of fetal EA $(13,14)$.

The present study has certain limitations. The study population was relatively small and localized, so the results may differ from those obtained in other populations or study settings. However, the thorough statistical analysis of the diagnostic accuracy of prenatal ultrasonography, the longitudinal nature of the study, and the use of postnatal examination as a gold standard are added strengths that improve its generalizability. Further studies in other settings or regions are necessary to confirm the findings of the present study. In addition, large-scale studies are required to correctly determine the diagnostic accuracy and role of prenatal ultrasonography in patients with EA/TOF.

The findings of the present study expand the limited information available regarding the diagnostic accuracy of prenatal ultrasonography and its application to fetuses with suspected EA/TOF. The findings are also useful for informing clinicians about the accuracy of prenatal ultrasonography for the screening and early diagnosis of EA/TOF during the fetal stages. The information obtained from a prenatal ultrasound may help clinicians to formulate a plan for the management of fetuses with suspected EA/TOF. However, since the results indicate that prenatal ultrasonography lacks a strong agreement with 
postnatal examinations and provides low diagnostic accuracy, it is recommended that clinicians should explore other diagnostic techniques to improve the accuracy of early diagnoses.

In conclusion, the results of the present study indicate that preoperative ultrasound has poor sensitivity but very good specificity for the diagnosis of EA/TOF. The use of ultrasound alone would result in a high rate of a false-positive diagnoses. However, it may be used as a preliminary screening tool to exclude patients for suspected EA/TOF.

\section{Acknowledgements}

Not applicable.

\section{Funding}

Funding was provided by Shandong Province Traditional Chinese Medicine Science and Technology Development Plan Project (grant no. 2019-0478) and Jining Key R\&D Plan Project (grant no. 2019SMNS004).

\section{Availability of data and materials}

The datasets used and/or analyzed during the current study are available from the corresponding author on reasonable request.

\section{Authors' contributions}

$\mathrm{CW}$ and YD contributed to the conception and design of the study. XN, ZZ and SW contributed to the acquisition, analysis and interpretation of the data. $\mathrm{CW}$ and YD confirmed the authenticity of all the raw data. CW drafted the manuscript and YD revised the paper. All authors read and approved the final manuscript.

\section{Ethics approval and consent to participate}

The Ethics Committee of the Affiliated Hospital of Jining Medical University approved the study protocol (approval no. JYFY-2019-17). All pregnant women provided informed consent.

\section{Patient consent for publication}

Not applicable.

\section{Competing interests}

The authors declare that they have no competing interests.

\section{References}

1. Marseglia L, Manti S, D'Angelo G, Gitto E, Salpietro C, Centorrino A, Scalfari G, Santoro G, Impellizzeri P and Romeo C: Gastroesophageal reflux and congenital gastrointestinal malformations. World J Gastroenterol 21: 8508-8515, 2015.

2. Pinheiro PFM, Simões e Silva AC and Pereira RM: Current knowledge on esophageal atresia. World J Gastroenterol 18: 3662-3672, 2012

3. Nassar N, Leoncini E, Amar E, Arteaga-Vázquez J, Bakker MK, Bower C, Canfield MA, Castilla EE, Cocchi G, Correa A, et al: Prevalence of esophageal atresia among 18 international birth defects surveillance programs. Birth Defects Res A Clin Mol Teratol 94: 893-899, 2012

4. Tandon RK, Sharma S, Sinha SK, Rashid KA, Dube R, Kureel SN, Wakhlu A and Rawat JD: Esophageal atresia: Factors influencing survival-experience at an Indian tertiary centre. J Indian Assoc Pediatr Surg 13: 2-6, 2008

5. Seo J, Kim DY, Kim AR, Kim DY, Kim SC, Kim IK, Kim KS, Yoon CH and Pi SY: An 18-year experience of tracheoesophageal fistula and esophageal atresia. Korean J Pediatr 53: 705-710, 2010.

6. Valevičienė NR, Varytė G, Zakarevičienė J, Kontrimavičiūtė E, Ramašauskaitè D and Rutkauskaitè-Valančienè D: Use of magnetic resonance imaging in evaluating fetal brain and abdomen malformations during pregnancy. Medicina (Kaunas) 55: 55, 2019.

7. Pardy C, D'Antonio F, Khalil A and Giuliani S: Prenatal detection of esophageal atresia: A systematic review and meta-analysis. Acta Obstet Gynecol Scand 98: 689-699, 2019.

8. Spitz L: Esophageal atresia. Lessons I have learned in a 40-year experience. J Pediatr Surg 41: 1635-1640, 2006.

9. Hands LJ and Dudley NE: A comparison between gap-length and Waterston classification as guides to mortality and morbidity after surgery for esophageal atresia. J Pediatr Surg 21: 404-406, 1986.

10. Bradshaw CJ, Thakkar H, Knutzen L, Marsh R, Pacilli M, Impey L and Lakhoo K: Accuracy of prenatal detection of tracheoesophageal fistula and oesophageal atresia. J Pediatr Surg 51: 1268-1272, 2016

11. Su P, Yuan Y, Zhang Z, Huang Y and Wang W: Application of high-frequency ultrasound in esophageal atresia with distal fistula. Dis Esophagus 27: 325-329, 2014.

12. Langer J, Hussain H, Khan A, Minkes R, Gray D, Siegel M and Ryan G: Prenatal diagnosis of esophageal atresia using sonography and magnetic resonance imaging. J Pediatr Surg 36: 804-807, 2001.

13. Spaggiari E, Faure G, Rousseau V, Sonigo $P$, Millischer-Bellaiche AE, Kermorvant-Duchemin E, Muller F, Czerkiewicz I, Ville Y and Salomon LJ: Performance of prenatal diagnosis in esophageal atresia. Prenat Diagn 35: 888-893, 2015.

14. Choudhry M, Boyd PA, Chamberlain PF and Lakhoo K: Prenatal diagnosis of tracheo-oesophageal fistula and oesophageal atresia. Prenat Diagn 27: 608-610, 2007. 